\title{
The clinical significance of antinuclear antibodies and specific autoantibodies in juvenile and adult systemic lupus erythematosus patients
}

\author{
Pongsawat Rodsaward, ${ }^{1}$ Natcha Chottawornsak, ${ }^{2}$ Supaporn Suwanchote, ${ }^{1}$ Muanpetch Rachayon, ${ }^{1}$ Tawatchai Deekajorndech, ${ }^{4}$ \\ Helen L Wright, ${ }^{5}$ Steven W Edwards, ${ }^{6}$ Michael W Beresford, ${ }^{7,8}$ Pawinee Rerknimitr, ${ }^{2,3}$ Direkrit Chiewchengchol ${ }^{1,3}$
}

\begin{abstract}
Background: Juvenile systemic lupus erythematosus (JSLE) and adult SLE (ASLE) patients present with different clinical manifestations, but it is unknown if there are differences in their antinuclear autoantibody (ANA) profiles or if staining patterns are associated with specific autoantibodies and clinical manifestations.
\end{abstract}

Objective: To determine whether distinct types and numbers of ANA-staining patterns are associated with specific autoantibodies and clinical manifestations in JSLE and ASLE patients.

Methods: A retrospective study was performed in Thai children $(\mathrm{n}=146)$ and adults $(\mathrm{n}=180)$ diagnosed with SLE using the Systemic Lupus International Collaborating Clinics classification criteria.

Results: JSLE patients with a homogeneous pattern of staining and anti-dsDNA or anti-nucleosome antibodies in serum, developed renal involvement, leukopenia and acute/subacute cutaneous LE. Coarse speckled pattern with anti-RNP or anti-Sm showed thrombocytopenia and renal involvement in JSLE patients, but leukopenia in both groups. JSLE patients with fine-coarse speckled pattern and anti-RNP, anti-Sm, anti-Ro-52 or anti-SSA developed leukopenia, thrombocytopenia and renal involvement, whilst hemolytic anemia and serositis were commonly found in those with anti-Ro-52. Median SLEDAI score was higher in JSLE than ASLE patients.

Conclusion: Detailed ANA-staining patterns with specific autoantibodies show particular clinical manifestations and hence prompt further clinical investigations in both JSLE and ASLE patients. Therefore, this study demonstrates that distinct patterns of ANA staining and specific autoantibodies are clinically important in both children and adults with SLE.

Key words: antinuclear antibodies, systemic lupus erythematosus, autoantibodies, autoimmune diseases, anti-double stranded DNA

\section{Affiliations:}

${ }^{1}$ Center of Excellence in Immunology and Immune-mediated Diseases, Department of Microbiology;

${ }^{2}$ Division of Dermatology,

${ }^{3}$ Skin and Allergy Research Unit, Department of Medicine;

${ }^{4}$ Division of Nephrology, Department of Pediatrics,

Faculty of Medicine, Chulalongkorn University, Bangkok, Thailand;

${ }^{5}$ Institute of Ageing and Chronic Disease;

${ }^{6}$ Institute of Integrative Biology;

Institute of Translational Medicine, University of Liverpool, Liverpool, United Kingdom

${ }^{8}$ Department of Pediatric Rheumatology, Alder Hey Children's NHS Foundation Trust, Liverpool, United Kingdom.
Corresponding author:

Direkrit Chiewchengchol

Center of Excellence in Immunology and Immune-mediated Diseases,

Faculty of Medicine, Chulalongkorn University, Rama 4 Road,

Pathumwan, Bangkok, Thailand, 10330

E-mail: cdirekrit@live.com

\section{Introduction}

Antinuclear antibodies (ANA) are serum autoantibodies directed against nuclear cell antigens. They are produced by auto-reactive B lymphocytes and are commonly found in patients with autoimmune diseases. It is believed that abnormal ANA production leads to formation of antigen-antibody complexes which are implicated in the pathogenesis of 
autoimmune diseases. ${ }^{1,2}$ Systemic lupus erythematosus (SLE) is one of the most common autoimmune diseases in both children and adults, and diagnosis is made in accordance with the revised classification criteria of the American College of Rheumatology (ACR) 1997. ${ }^{3}$ However, the new Systemic Lupus International Collaborating Clinics (SLICC) group classification criteria has been introduced and is now widely used. ${ }^{4}$ Notably, the presence of ANA in serum is one of the immunological criteria for the diagnosis of SLE in both the ACR 1997 criteria and the new SLICC group classification criteria.

Serum ANA are usually detected by indirect immunofluorescence (IIF) techniques and quantifies both the ANA titers and their staining patterns. ${ }^{5}$ ANA titers above $1: 80$ are considered as a positive result, ${ }^{6-8}$ and high titers $(\geq 1: 640)$ are suggestive of SLE, particularly when other clinical manifestations are observed. ${ }^{9}$ However, ANA titers are not correlated with disease activity and severity. ${ }^{10}$ ANA-staining patterns of SLE patient serum are normally identified in a cell line from epidermoid carcinoma of the larynx (Hep-2 cell) fixed on a slide and visualized using IIF techniques. ${ }^{11}$ The International Consensus on ANA-staining Patterns (ICAP) classifies patterns into 3 main types; nuclear (e.g. homogeneous, fine speckled, coarse speckled, etc.), cytoplasmic and mitotic. ${ }^{12}$ As there are many possible nuclear antigens, ANA are classified into specific autoantibodies using different techniques such as immunoblotting or enzyme-linked immunosorbent assay; for example, anti-dsDNA, anti-Sm, anti-RNP, anti-SSA/Ro, anti-Ro-52, anti-SSB/La, anti-nucleosome, etc. ${ }^{13}$ It has been reported that some patterns of ANA staining are associated with certain nuclear antigens that are related to particular manifestations of the disease. ${ }^{14}$

There are only a few reports demonstrating the clinical significance of ANA-staining patterns in SLE patients particularly those who are diagnosed by the new SLICC classification criteria. Moreover, the clinical manifestations in juvenile SLE (JSLE) are more severe than adult SLE (ASLE) but it is unknown if there are distinct ANA-staining patterns between the two groups. ${ }^{15-17}$ In this study, we therefore sought to investigate whether ANA-staining patterns and number of patterns at diagnosis could predict specific autoantibodies, clinical involvement and disease activity in JSLE compared with the adult counterparts.

\section{Methods}

We performed a retrospective, descriptive study of Thai JSLE patients ( $<18$ years old) and ASLE patients who attended clinics at the King Chulalongkorn Memorial Hospital from 1 January 2015 to 31 December 2016. All SLE patients were treated as outpatients (both pediatric and adult who attended dermatology, nephrology, rheumatology, hematology and other clinics) or as inpatient. These patients were identified using ICD-10 with the codes of M32.1 (Systemic lupus erythematosus with organ or system involvement), M32.8 (Other forms of systemic lupus erythematosus) and M32.9 (Systemic lupus erythematosus, unspecified). The inclusion criteria were Asian-Thai children or adults who were diagnosed with SLE by SLICC criteria. The exclusion criteria were patients with unknown or negative ANA test.
Patient medical records/charts, SLE disease activity index (SLEDAI) scores and laboratory results including ANA and specific autoantibodies at diagnosis were reviewed. ${ }^{18}$

\section{Detection of antinuclear antibodies (ANA)}

Serum ANA titers and ANA-staining patterns were detected by IIF. Each patient serum sample was initially diluted at 1:80 and 1:640 (and later at 2-fold dilutions) and added onto Hep-2 cells fixed on a slide (EUROIMMUN IF-Spinter, Germany). The formation of nuclear antigen-autoantibody complexes was detected by staining with anti-IgG antibody tagged with Propidium Iodide and visualized under fluorescence microscopy (EUROPattern microscope, Germany). The ANA titers were scored as negative $(\leq 1: 80)$, or positive (ranging from $1: 160$ to $\geq 1: 2,560)$. The staining patterns were identified using a EUROPattern microscope once ANA titers were identified as positive.

\section{Detection of specific autoantibodies}

Specific autoantibodies in patient sera were detected by Immunoblotting (EUROIMMUN, Germany). Serum was initially diluted at 1:100 and added into a Immunostrip coated with 12 different specific nuclear antigens (dsDNA, Sm, RNP, SS-A, SS-B, Ro-52, nucleosome, histone, centromere, Scl-70, Jo-1 and ribosomal P). After specific nuclear antigen-antibody complexes were formed, enzyme-labelled anti-human IgG antibody was added to conjugate with the complexes. The Immunostrip was finally incubated with chromogen substrate and then analyzed by a EurolineScan (Germany).

\section{Ethical approval}

Ethical approval was obtained from The Ethics of Institutional Review Board (IRB) from Faculty of Medicine, Chulalongkorn University prior to commencing the study (IRB No. 309/60), and The Thai Clinical Trials Registry (TCTR 20170928002)

(http://www.clinicaltrials.in.th/index.php?tp=regtrials\&menu= trialsearch\&smenu $=$ fulltext $\&$ task $=$ search \&task $2=$ view $1 \& \mathrm{id}=$ $\underline{2860})$

\section{Statistical analyses}

Statistical analyses were performed using SPSS version 22 software. The associations between ANA-staining patterns and specific autoantibodies, and ANA-staining patterns/specific autoantibodies and clinical manifestations were analyzed using Pearson's Chi-squared test. The association between the number of ANA-staining patterns and disease activity were analyzed using Pearson correlation coefficient. $P$-values $<0.05$ were considered significant.

\section{Results \\ Demographic data}

A total of 377 patients (171 children and 206 adults) diagnosed with SLE using the SLICC diagnostic criteria were recruited into our study. Four patients with negative ANA and 47 patients with unknown ANA status were excluded from the study. Of the 326 SLE patients included in the study, $146(44.7 \%)$ were JSLE and 180 (55.2\%) were ASLE patients. 
The ratios of females to males was 8.7:1 and 9.6:1 in JSLE and ASLE, respectively. The ethnicity of both JSLE and ASLE were all Asian-Thai. The mean age at diagnosis was 11.7 years $( \pm 3.7)$ in JSLE and 33.2 years $( \pm 10.2)$ in ASLE patients. The mean duration of follow up was approximately 7 years in both groups (JSLE; 3.4-11 years and ASLE; 3.2-13 years). The median SLEDAI score at diagnosis was 14 (IQ range: 8-18) in JSLE but lower in ASLE patients (SLEDAI = 10, with IQ range: 6-14).

\section{Antinuclear antibodies (ANA) \\ ANA titers and staining patterns}

The majority of SLE patients (> 98\%) showed a positive serum ANA result and $48.8 \%$ of the patients $(n=159)$ showed serum ANA titers $\geq 1: 2,560$. The staining patterns in JSLE and ASLE at diagnosis are summarized in Figure 1. Homogeneous staining pattern was the most common type in both groups but more common in JSLE patients (71.9\% VS 56.6\%). The percentages of fine speckled and coarse speckled staining patterns in JSLE and ASLE patients were comparable (fine speckled: $47.2 \%$ VS $43.3 \%$ and coarse speckled: $24.6 \%$ VS $25 \%$, respectively). Nuclear envelope and cytoplasmic staining patterns were infrequently seen in both groups $(\sim 10 \%)$. Other staining patterns, including centromere and PCNA-like patterns were rarely found and only observed in ASLE patients.

\section{Number of ANA-staining patterns}

The numbers of ANA-staining patterns at diagnosis are summarized in Table 1. Single ANA-staining pattern was found in $38 \%$ of JSLE patients and homogeneous pattern was the most common type (75\%), followed by fine speckled (11\%) and coarse speckled (7\%) patterns. Two ANA-staining patterns were found in $47 \%$ of JSLE patients and homogeneous-fine speckled (41\%) pattern was the most common finding, followed by fine-coarse speckled (22\%) and homogeneous-nuclear envelope (12\%) patterns, respectively.
In ASLE, single ANA-staining pattern was found in 44\% of the patients. The homogeneous pattern was the most common type (51\%), followed by fine speckled (16\%) and coarse speckled (5\%) patterns, similar to the juvenile group. Adult patients (42\%) showed two ANA-staining patterns, which were fine-coarse speckled (29\%), homogeneous-fine speckled (23\%), homogeneous-nuclear envelope (11\%) patterns whilst other patterns were less common. Three or more patterns were rarely seen in both groups (e.g. fine-coarse speckled-nuclear dot patterns).

Table 1. The number and type of ANA-staining patterns in JSLE $(n=146)$ and ASLE $(n=180)$ patients

\begin{tabular}{|lcc|}
\hline \multicolumn{1}{|c}{ Number or pattern } & $\begin{array}{c}\text { Number } \\
\text { of JSLE } \\
(\%)\end{array}$ & $\begin{array}{c}\text { Number } \\
\text { of ASLE } \\
(\%)\end{array}$ \\
\hline 1. Single pattern & $56(38 \%)$ & $79(44 \%)$ \\
\hline Homogeneous & $42(75 \%)$ & $40(51 \%)$ \\
\hline Fine speckled & $6(11 \%)$ & $13(16 \%)$ \\
\hline Coarse speckled & $4(7 \%)$ & $4(5 \%)$ \\
\hline Nuclear envelope & - & $2(2.5 \%)$ \\
\hline PCNA-like & - & $2(2.5 \%)$ \\
\hline Others & $4(7 \%)$ & $18(23 \%)$ \\
\hline Two patterns & $68(47 \%)$ & $75(42 \%)$ \\
\hline Homogeneous-fine speckled & $28(41 \%)$ & $17(23 \%)$ \\
\hline Fine-coarse speckled & $14(22 \%)$ & $22(29 \%)$ \\
\hline Homogeneous-nuclear envelope & $8(21 \%)$ & $5(25 \%)$ \\
\hline Fthers & $18(79 \%)$ & $15(75 \%)$ \\
\hline Four patterns or more & $8(11 \%)$ & $6(3 \%)$ \\
\hline
\end{tabular}

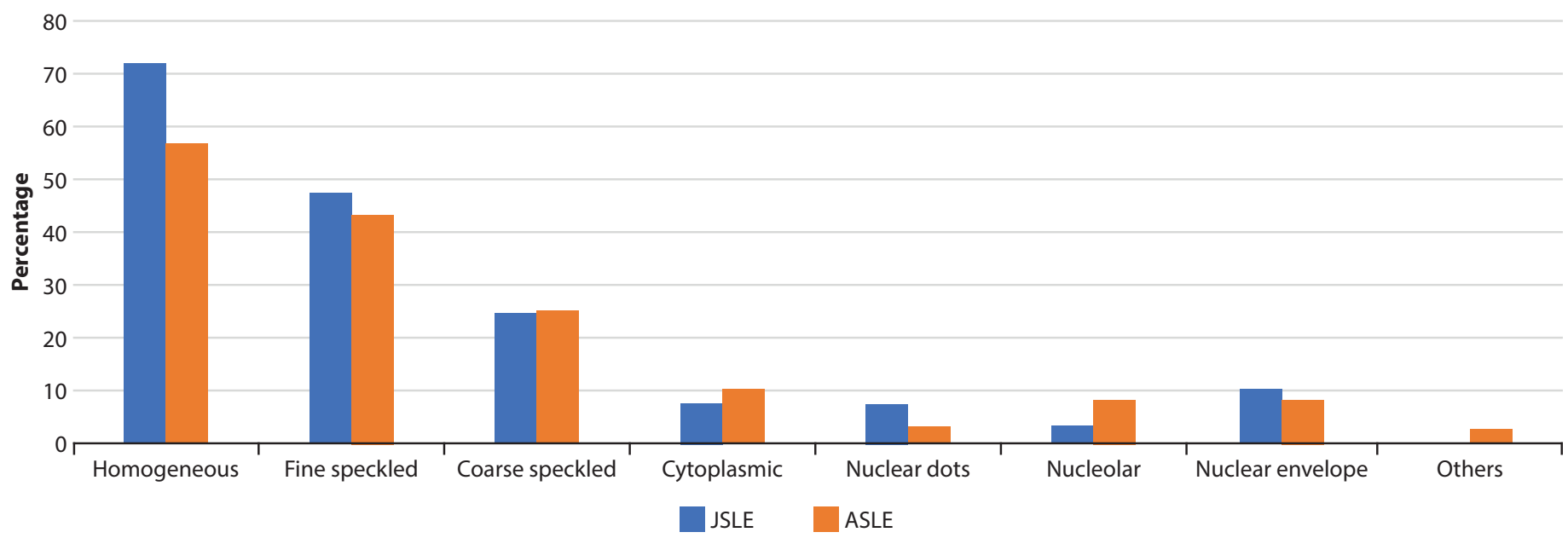

Figure 1. The percentages of ANA-staining patterns in JSLE $(n=146)$ and ASLE $(n=180)$ patients. 


\section{ANA-staining patterns VS specific autoantibodies}

The association between ANA-staining patterns and specific autoantibodies was analyzed in the serum of 85 SLE patients (59 children and 26 adults) and summarized in Table 2. The results demonstrated a positive association between homogeneous pattern in JSLE patients, and anti-dsDNA and anti-nucleosome $(p$-value $<0.001)$ with the odds ratio of 21 $(95 \% \mathrm{CI}=4.2-104.8)$ and $8.4(95 \% \mathrm{CI}=2.3-30.1)$, respectively. Coarse speckled pattern showed a positive association with anti-RNP and anti-Sm in both JSLE and ASLE patients ( $p$-value $<0.05)$. The association between coarse speckled pattern and anti-RNP showed an odds ratio of 10 in both JSLE and ASLE patients $(95 \% \mathrm{CI}=2.8-35.2$ and 1.4-69.3, respectively) whereas the association between coarse speckled pattern and anti-Sm showed an odds ratio of $9(95 \% \mathrm{CI}=2.4-34.3)$ in JSLE and $22(95 \% \mathrm{CI}=2.1-236.1)$ in ASLE patients.

In SLE patients with two ANA-staining patterns, there was only a positive association between fine-coarse speckled pattern and anti-RNP, anti-Sm, anti-Ro-52 and anti-SSA ( $p$-value $<0.05$ ) with the odd ratios of up to 5 in JSLE patients.

\section{ANA-staining patterns with specific autoantibodies VS clini- cal involvement}

In line with the new SLICC classification criteria, 11 clinical criteria in SLE patients with particular ANA-staining patterns and specific autoantibodies in described Table 2 were explored and summarized in Table 3 . The results showed that JSLE patients who had homogeneous staining pattern with either anti-dsDNA or anti-nucleosome in their serum developed renal involvement (> 75\%), leukopenia (> 60\%) and acute or subacute cutaneous LE $(>60 \%)$ during the follow-up period. More than $58 \%$ of JSLE patients who had coarse speckled pattern with either anti-RNP or anti-Sm developed leukopenia, thrombocytopenia and renal involvement: these clinical involvements were found in $>62 \%$ of JSLE patients who had fine-coarse speckled pattern with either anti-RNP or anti-Sm or anti-SSA. Furthermore, fine-coarse speckled pattern and anti-Ro-52 in JSLE patients were related to hemolytic anemia and serositis (> 57\%).

In ASLE, $>60 \%$ of patients who had coarse speckled pattern with either anti-RNP or anti-Sm in their serum developed leukopenia during the follow-up period.

Table 2. The significant associations between ANA-staining patterns and autoantibodies in JSLE and ASLE patients

\begin{tabular}{|c|c|c|c|c|c|c|}
\hline ANA patterns & Total number & $\begin{array}{c}\text { Types of } \\
\text { Autoantibodies }\end{array}$ & $\begin{array}{l}\text { Number of patients with positive } \\
\text { autoantibody per total (\%) }\end{array}$ & $p$-value & $\begin{array}{l}\text { Odds } \\
\text { ratio }\end{array}$ & $\begin{array}{l}\text { 95\% Confidence } \\
\text { Interval }\end{array}$ \\
\hline \multirow[t]{2}{*}{ 1. Homogeneous } & JSLE $(n=36)$ & dsDNA & $24 / 36(67 \%)$ & $<0.001$ & 21 & $4.2-104.8$ \\
\hline & & nucleosome & $23 / 36(64 \%)$ & 0.001 & 8.4 & $2.3-30.1$ \\
\hline \multirow[t]{4}{*}{ 2. Coarse-speckled } & JSLE $(\mathrm{n}=22)$ & RNP & $15 / 22(68 \%)$ & $<0.001$ & 10 & $2.8-35.2$ \\
\hline & & $\mathrm{Sm}$ & $12 / 22(55 \%)$ & 0.001 & 9 & $2.4-34.3$ \\
\hline & Adult SLE $(\mathrm{n}=12)$ & RNP & $10 / 12(83 \%)$ & 0.036 & 10 & $1.4-69.3$ \\
\hline & & Sm & $8 / 12(67 \%)$ & 0.009 & 22 & $2.1-236.1$ \\
\hline \multirow[t]{4}{*}{ 3. Fine-coarse speckled } & JSLE $(\mathrm{n}=12)$ & RNP & $8 / 12(67 \%)$ & 0.04 & 4.8 & $1.2-18.7$ \\
\hline & & $\mathrm{Sm}$ & $7 / 12(58 \%)$ & 0.01 & 5.4 & $1.4-21.2$ \\
\hline & & Ro-52 & 7/12 (58\%) & 0.028 & 4.2 & $1.1-16$ \\
\hline & & SSA & $8 / 12(67 \%)$ & 0.018 & 5.3 & $1.4-21$ \\
\hline
\end{tabular}

Table 3. The clinical involvements in JSLE and ASLE patients with particular ANA-staining patterns and specific autoantibodies, according to the new SLICC classification criteria ${ }^{4}$

\begin{tabular}{llr} 
1. JSLE with homogeneous pattern $(\mathbf{N}=\mathbf{3 6})$ & Clinical involvement & \% of patients \\
\hline 1.1 Anti-dsDNA & Renal involvement & 75 \\
& Leukopenia & 62.5 \\
& Acute or Subacute cutaneous LE & 62.5 \\
& Others & $<50$ \\
\hline 1.2 Anti-nucleosome & Renal involvement & 82.6 \\
& Leukopenia & 60.8 \\
\hline & Acute or Subacute cutaneous LE & 60.8 \\
\hline & Others & $<50$ \\
\hline
\end{tabular}




\section{Table 3. (Continued)}

\begin{tabular}{|c|c|c|}
\hline 2. JSLE with coarse speckled pattern $(\mathrm{N}=22)$ & Clinical involvement & $\%$ of patients \\
\hline \multirow[t]{5}{*}{ 2.1 Anti-RNP } & Renal involvement & 66.6 \\
\hline & Leukopenia & 66.6 \\
\hline & Thrombocytopenia & 60 \\
\hline & Acute or Subacute cutaneous LE & 53.3 \\
\hline & Others & $<50$ \\
\hline \multirow[t]{5}{*}{ 2.2 Anti-Sm } & Acute or Subacute cutaneous LE & 66.6 \\
\hline & Renal involvement & 58.3 \\
\hline & Leukopenia & 58.3 \\
\hline & Thrombocytopenia & 58.3 \\
\hline & Others & $<50$ \\
\hline 3. JSLE with fine-coarse speckled pattern $(\mathrm{N}=12)$ & Clinical involvement & $\%$ of patients \\
\hline \multirow[t]{4}{*}{ 3.1 Anti-RNP } & Leukopenia & 75 \\
\hline & Renal & 62.5 \\
\hline & Thrombocytopenia & 62.5 \\
\hline & Others & $<50$ \\
\hline \multirow[t]{4}{*}{ 3.2 Anti-Sm } & Leukopenia & 71.4 \\
\hline & Thrombocytopenia & 71.4 \\
\hline & Renal & 57.1 \\
\hline & Others & $<50$ \\
\hline \multirow[t]{5}{*}{ 3.3 Anti-Ro-52 } & Renal involvement & 85.7 \\
\hline & Hemolytic anemia & 71.4 \\
\hline & Serositis & 57.1 \\
\hline & Leukopenia & 57.1 \\
\hline & Others & $<50$ \\
\hline \multirow[t]{3}{*}{ 3.4 Anti-SSA } & Leukopenia & 75 \\
\hline & Renal involvement & 62.5 \\
\hline & Others & $<50$ \\
\hline 4. ASLE with coarse speckled pattern $(N=12)$ & Clinical involvement & $\%$ of patients \\
\hline \multirow[t]{2}{*}{ 4.1 Anti-RNP } & Leukopenia & 60 \\
\hline & Others & $<50$ \\
\hline \multirow[t]{2}{*}{ 4.2 Anti-Sm } & Leukopenia & 62.5 \\
\hline & Others & $<50$ \\
\hline
\end{tabular}




\section{Number of ANA-staining patterns VS disease severity}

The association between the number of ANA-staining patterns and disease activity using SLEDAI scores was analyzed. The results showed that the median SLEDAI score in JSLE patients was 14 across the number of patterns, whereas the median score in ASLE patients gradually rose from 10 to 19 once the number of patterns was increased from one to four (Figure 2), but this did not reach statistical significance in either group ( $p$-value $=0.846$ and 0.055 , respectively).

\section{A}

50

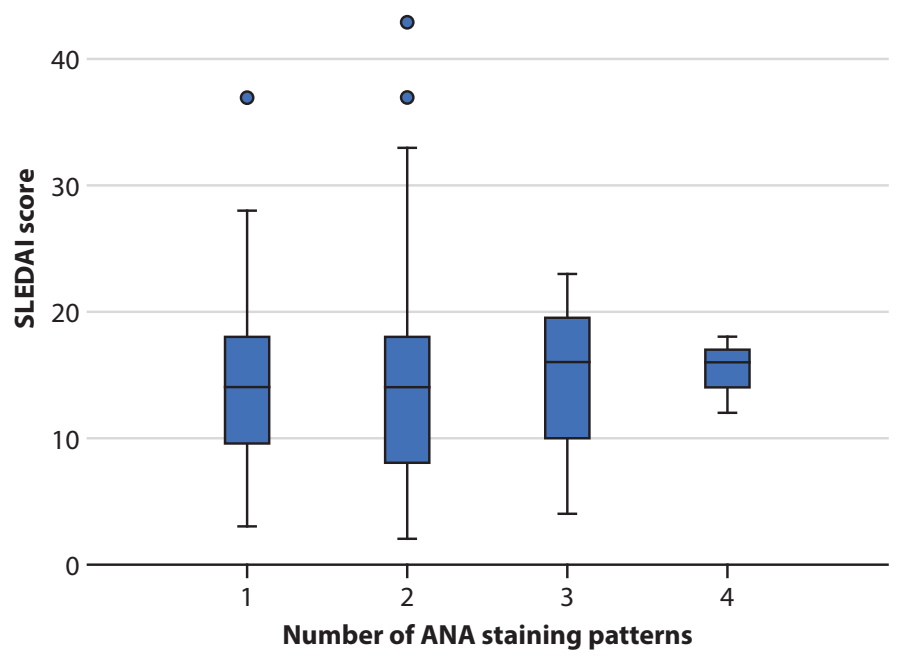

B

50$$
40
$$

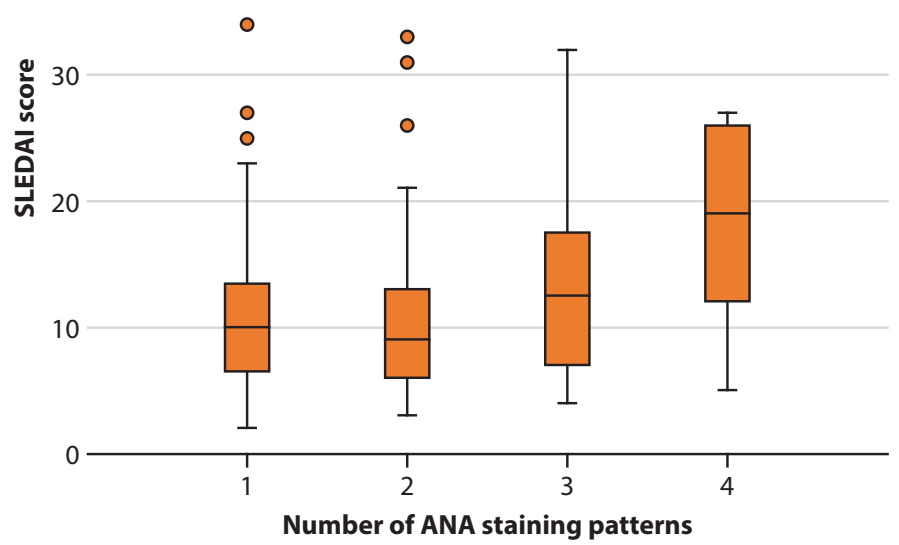

Figure 2. The association between numbers of ANA-staining patterns and severity scores (SLEDAI) in 146 JSLE (A) patients, and 180 ASLE (B) patients.

\section{Discussion}

The presence of ANA in serum is one of the key features in the SLICC classification criteria for making a diagnosis of SLE. ${ }^{4}$ IIF is a routine technique used for testing ANA in patient serum and its sensitivity and specificity make it a highly suitable and robust technique for this diagnosis. ${ }^{5}$ In our study, the majority of patients with SLE (> 98\%) showed a positive serum ANA result and the ANA-staining patterns were similar in both children and adults with SLE. The most common staining types were homogenous pattern in both groups, followed by fine and coarse speckled patterns, respectively, which are consistent with previous studies. ${ }^{16,17,19,20}$ Interestingly, our study demonstrated that almost 50\% of JSLE (47\%) and ASLE (42\%) patients had developed two ANA-staining patterns in their serum at diagnosis, and fine-coarse speckled and homogeneous-fine speckled patterns were the most prominent findings in both groups. This finding has been rarely reported; therefore, we further investigated the importance of these two ANA-staining patterns in terms of types of autoantibodies and association with different clinical manifestations.

Although most patients with SLE (48.8\%) showed very high ANA titers $(>1: 2,560)$ in their serum, it has been reported that titers are not correlated with disease activity and/ or severity. ${ }^{10}$ However, a previous report has suggested that ANA-staining patterns are associated with specific autoantibodies, ${ }^{12}$ that are probably related to particular clinical symptoms. For example, SLE patients with homogeneous pattern usually develop anti-dsDNA and anti-nucleosome in their serum. ${ }^{21}$ This finding is similar to our study which showed a positive association between homogeneous staining pattern and anti-dsDNA or anti-nucleosome antibodies. Therefore, our findings suggest that homogeneous staining pattern, the most common pattern in SLE patients, is associated with anti-dsDNA and anti-nucleosome antibodies, particularly in JSLE patients. These associations were also found in ASLE patients in our study but they did not reach statistical significance ( $\mathrm{p}=0.648$ and 0.216 ), probably due to a limitation that only 26 ASLE patients were tested for specific autoantibodies.

Furthermore, our results showed that coarse speckled staining pattern or coarse speckled staining in combination with fine speckled patterns were associated with anti-RNP and anti-Sm in JSLE and ASLE patients, which is consistent with a previous study. ${ }^{12}$ However, an association between fine speckled pattern and anti SS-A or anti SS-B as previously reported ${ }^{12}$ was not found in our studies, probably because of differences in populations and techniques used for detection.

The clinical manifestations of SLE are varied in both children and adults. ${ }^{22}$ Previous studies reported that some autoantibodies were associated with particular organ or system involvements. ${ }^{23-25}$ For instance, anti-dsDNA was associated with renal involvement ${ }^{23}$ whereas anti-SS-A/Ro was associated with subacute cutaneous $\mathrm{LE}^{26}$ Therefore, this study further investigated the clinical involvements based on 11 clinical criteria listed in the new SLICC classification in those SLE patients who had particular ANA-staining pattern with specific autoantibodies shown in Table 2. Expectedly, renal involvement was commonly found in JSLE patients who had homogeneous pattern with anti-dsDNA in their serum which was consistent with previous studies. ${ }^{14}$ However, our study demonstrated 
that renal involvement was also found in JSLE patients with homogeneous and anti-nucleosome autoantibodies in their serum. Furthermore, more than half of JSLE patients with homogeneous staining pattern and anti-dsDNA or anti-nucleosome developed leukopenia and acute or subacute cutaneous LE, suggesting that JSLE patients with these particular patterns and specific autoantibodies are probably at risk of leukopenia, renal and cutaneous involvements.

A new finding from our study was that more than $60 \%$ of JSLE patients who had coarse or fine-coarse speckled staining patterns with either anti-RNP, anti-Sm or anti-SSA developed leukopenia, thrombocytopenia and renal involvement. These findings suggest that JSLE patients with these particular ANA patterns and specific autoantibodies should be investigated for hematological and renal involvements. Furthermore, serositis and hemolytic anemia should be further investigated in JSLE patients who had fine-coarse speckled pattern with anti-Ro-52, as our study found that more than $57 \%$ of these JSLE patients developed those symptoms during the follow-up period. However, in adult patients, more than $60 \%$ of those who had coarse speckled staining pattern with anti-RNP or anti-Sm in their serum developed leukopenia, suggesting that hematological analyses should be investigated in these adult patients.

Further statistical analyses of the associations between ANA staining patterns/specific autoantibodies and clinical involvements were performed to confirm these observations. However, the results showed insignificant differences ( $p$ $>0.05$ ), probably due to the small sample size which was a limitation of this study: while our findings suggest a trend of these associations, a future (cohort) study with a larger sample size is required.

As more than half of SLE patients showed two or more ANA-staining patterns, and distinct ANA patterns were associated with specific autoantibodies and particular clinical involvements, we hypothesized that SLE patients with more ANA-staining patterns could develop multiple organ involvements which probably reflected disease activity and severity. Therefore, we investigated whether there was any association between the number of ANA-staining patterns and disease activity using SLEDAI scores. The results showed that the overall SLEDAI score in ASLE patients was lower than in children with SLE (10 VS 14). This finding is consistent with previous studies demonstrating that JSLE patients have clinically more severe disease than ASLE patients. ${ }^{27}$ Interestingly, a higher number of ANA-staining patterns $(>2$ ) tended to correlate with disease activity, particularly in ASLE patients (Figure 2), and also those adults with 4 patterns showed the highest activity (median SLEDAI = 19). However, these observations were not statistically significant.

In conclusion, this is the first Thai JSLE and ASLE retrospective study that includes a large number of patients and demonstrates ANA-staining patterns and number of patterns in children and adults. Our study proposes that ANA-staining patterns and specific autoantibodies in SLE patients are important to indicate clinical involvement. JSLE patients with homogeneous pattern and anti-dsDNA or anti-nucleosome usually develop renal involvement and leukopenia, and acute or subacute cutaneous LE, whilst ASLE patients with fine-coarse speckled pattern and anti-RNP or anti-Sm may develop leukopenia. JSLE patients with fine speckled or finecoarse speckled patterns who have anti-RNP, anti-Sm, anti-Ro-52 or anti-SSA should be investigated for renal and hematological involvements, including serositis and hemolytic anemia particularly those with anti-Ro-52. The clinical involvements in ASLE are mostly milder than JSLE patients and number of ANA patterns shows no significant differences in disease activity in both groups.

\section{Key messages}

- JSLE patients with homogeneous staining pattern and anti-dsDNA or anti-nucleosome antibodies should be investigated for renal involvement, leukopenia and acute/subacute cutaneous LE.

- JSLE patients with coarse or fine-coarse speckled patterns with anti-RNP, anti-Sm, anti-Ro-52 or anti-SSA should be investigated for hematological or renal involvements.

- ASLE patients with fine-coarse speckled staining pattern with anti-RNP or anti-Sm should be investigated for leukopenia.

\section{Acknowledgement}

Special thanks go to the Center of Excellence in Immunology and Immune-mediated Diseases, Department of Microbiology, and the Skin and Allergy Research Unit, Department of Medicine, Faculty of Medicine, Chulalongkorn University, Bangkok, Thailand.

\section{Funding}

This was an unfunded study.

\section{Conflict of interest}

The authors have declared no conflicts of interest.

\section{References}

1. Reeves WH, Xu Y, Zhuang H, Li Y, Yang L. Origins of Antinuclear Antibodies. In: Lahita RG, editor. Systemic lupus erythematosus. 5th ed. London: Academic Press; 2011.p.213-33.

2. Pisetsky DS. Antinuclear antibody testing - misunderstood or misbegotten? Nat Rev Rheumatol. 2017;13(8):495-502.

3. Hochberg MC. Updating the American College of Rheumatology revised criteria for the classification of systemic lupus erythematosus. Arthritis Rheum. 1997;40(9):1725.

4. Petri M, Orbai AM, Alarcon GS, Gordon C, Merrill JT, Fortin PR, et al. Derivation and validation of the Systemic Lupus International Collaborating Clinics classification criteria for systemic lupus erythematosus. Arthritis Rheum. 2012;64(8):2677-86.

5. Meroni PL, Schur PH. ANA screening: an old test with new recommendations. Ann Rheum Dis. 2010;69(8):1420-2.

6. Tan EM, Feltkamp TE, Smolen JS, Butcher B, Dawkins R, Fritzler MJ, et al. Range of antinuclear antibodies in "healthy" individuals. Arthritis Rheum. 1997;40(9):1601-11.

7. Sack U, Conrad K, Csernok E, Frank I, Hiepe F, Krieger T, et al. Autoantibody detection using indirect immunofluorescence on HEp-2 cells. Ann N Y Acad Sci. 2009;1173:166-73.

8. Ghosh P, Dwivedi S, Naik S, Agarwal V, Verma A, Aggarwal A, et al Antinuclear antibodies by indirect immunofluorescence: optimum screening dilution for diagnosis of systemic lupus erythematosus. Indian J Med Res. 2007;126(1):34-8. 
9. Perilloux BC, Shetty AK, Leiva LE, Gedalia A. Antinuclear antibody (ANA) and ANA profile tests in children with autoimmune disorders: a retrospective study. Clin Rheumatol. 2000;19(3):200-3.

10. Satoh M, Chan EK, Sobel ES, Kimpel DL, Yamasaki Y, Narain S, et al. Clinical implication of autoantibodies in patients with systemic rheumatic diseases. Expert Rev Clin Immunol. 2007;3(5):721-38.

11. Hahon N, Eckert HL, Stewart J. Evaluation of cellular substrates for antinuclear antibody determinations. J Clin Microbiol. 1975;2(1):42-5.

12. Chan EK, Damoiseaux J, Carballo OG, Conrad K, de Melo Cruvinel W, Francescantonio PL, et al. Report of the First International Consensus on Standardized Nomenclature of Antinuclear Antibody HEp-2 Cell Patterns 2014-2015. Front Immunol. 2015;6:412.

13. Carter S, Carter J, Richardson D. The Euroimmun ANA Profile 1 as an Expanded ENA Profile. Am J Clin Pathol. 2014;142(suppl_1):A192.

14. Yung S, Chan TM. Molecular and Immunological Basis of Tubulo -Interstitial Injury in Lupus Nephritis: a Comprehensive Review. Clin Rev Allergy Immunol. 2017;52(2):149-63.

15. Almogren A. Anti-double stranded antibody. Association with titers and fluorescence patterns of anti-nuclear antibody in systemic lupus erythematosus. Saudi Med J. 2010;31(1):32-6.

16. Adelowo OO, Ojo O, Oduenyi I. Auto antibodies in Nigerian lupus patients. Afr J Med Med Sci. 2012;41(2):177-81.

17. Ahmed TA, Ikram N, Hussain T, Farooqui A, Haleem A, Bashir M, et al. Clinical and laboratory features of systemic lupus erythematosus (SLE) in Pakistani patients. J Pak Med Assoc. 2002;52(1):12-5.

18. Bombardier C, Gladman DD, Urowitz MB, Caron D, Chang CH. Derivation of the SLEDAI. A disease activity index for lupus patients. The Committee on Prognosis Studies in SLE. Arthritis Rheum. 1992;35(6): $630-40$.
19. Frodlund M, Dahlstrom O, Kastbom A, Skogh T, Sjowall C. Associations between antinuclear antibody staining patterns and clinical features of systemic lupus erythematosus: analysis of a regional Swedish register. BMJ Open. 2013;3(10):e003608.

20. Vila LM, Mayor AM, Valentin AH, Garcia-Soberal M, Vila S. Clinical and immunological manifestations in 134 Puerto Rican patients with systemic lupus erythematosus. Lupus. 1999;8(4):279-86.

21. Kumar Y, Bhatia A. Detection of antinuclear antibodies in SLE. Methods Mol Biol. 2014;1134:37-45.

22. Tsokos GC. Systemic lupus erythematosus. N Engl J Med. 2011;365(22): 2110-21.

23. Bossuyt X, Hendrickx A, Frans J. Antinuclear antibody titer and antibodies to extractable nuclear antigens. Arthritis Rheum. 2005;53(6):987-8.

24. Novak GV, Marques M, Balbi V, Gormezano NW, Kozu K, Sakamoto AP, et al. Anti-RO/SSA and anti-La/SSB antibodies: Association with mild lupus manifestations in 645 childhood-onset systemic lupus erythematosus. Autoimmun Rev. 2017;16(2):132-5.

25. Flechsig A, Rose T, Barkhudarova F, Strauss R, Klotsche J, Dahnrich C, et al. What is the clinical significance of anti-Sm antibodies in systemic lupus erythematosus? A comparison with anti-dsDNA antibodies and C3. Clin Exp Rheumatol. 2017; 35(4): 598-606.

26. Alniemi DT, Gutierrez A Jr, Drage LA, Wetter DA. Subacute Cutaneous Lupus Erythematosus: Clinical Characteristics, Disease Associations, Treatments, and Outcomes in a Series of 90 Patients at Mayo Clinic, 1996-2011. Mayo Clin Proc. 2017;92(3):406-14

27. Jimenez S, Cervera R, Font J, Ingelmo M. The epidemiology of systemic lupus erythematosus. Clin Rev Allergy Immunol. 2003;25(1):3-12. 